\section{Growth hormone therapy has a positive effect on cardiovascular risk}

Patients with growth hormone deficiency (GHD) have an increased risk of obesity, insulin resistance, lipid alterations, atherosclerotic changes, and also have reduced cardiac performance, all of which increase morbidity and mortality. In a placebo-controlled, double-blind, crossover study, Bollerslev et al. investigated the effects of $\mathrm{GH}$ therapy on cardiovascular risk factors in patients with GHD.

A total of 55 patients with severe, adult-onset GHD of at least 2 years' duration underwent consecutive 9-month periods of treatment with recombinant human GH therapy (Genotropin ${ }^{\circledR}$, Pfizer, New York, NY, USA) and placebo. There was a 4-month washout period in between treatments. The dose of GH therapy given to each patient was individually titrated to maintain levels of serum insulin-like growth factor 1 to within the normal range. Women were given a $50 \%$ higher mean dose of GH therapy than men, such that similar levels of serum insulin-like growth factor 1 were reached in both sexes. GH therapy decreased serum C-reactive protein by $41 \%$ and improved levels of apolipoprotein B cholesterol compared with placebo. There was no effect on levels of apolipoprotein A-1 and interleukin-6 in patients throughout the study period.

The authors conclude that, in treatment-naive patients with adult-onset GHD, GH-replacement therapy has a positive effect on cardiovascular risk, mediated by decreased levels of both apolipoprotein B and C-reactive protein. These factors are markers of atherogenesis and subclinical inflammation, respectively.

Original article Bollerslev J et al. (2006) Positive effects of a physiological dose of $\mathrm{GH}$ on markers of atherogenesis: a placebo-controlled study in patients with adult-onset GH deficiency. Eur J Endocrinol 154: 537-543

\section{Combining prestroke treatments reduces stroke severity}

Antiplatelet agents, angiotensin-convertingenzyme inhibitors and statins are well known to be effective in stroke prevention. A retrospective study by researchers at the Beth Israel Deaconess Medical Center has shown that, if these drugs are used in combination prior to stroke, stroke severity might be reduced further and the volume of ischemic tissue at risk decreased.

All patients in the study presented within $24 \mathrm{~h}$ of stroke onset. The 20 patients on all three stroke prevention therapies at stroke onset had a significantly lower NIS Stroke Scale score $(P=0.001)$ and smaller perfusion-diffusion mismatch lesion volumes-indicating smaller volumes of ischemic tissue at risk - than the 43 patients on an antiplatelet agent plus a statin or angiotensin-converting-enzyme inhibitor, the 47 patients on antiplatelets alone, and the 69 patients taking no stroke treatment. Patients taking triple therapy were also more likely to have a shorter length of hospital stay and better functional status upon discharge, although this was probably due to differences in the initial severity of stroke rather than differences in the effect of the treatments on recovery.

The authors state that the additive effects of the combined therapies are probably caused by their differing modes of action. The findings of this study are preliminary and cannot lead directly to a recommendation for triple therapy in all patients at risk for stroke, but they nevertheless provide an important basis for further investigation.

Original article Kumar S et al. (2006) Antiplatelets,

ACE inhibitors, and statins combination reduces stroke severity and tissue at risk. Neurology 66:

$1153-1158$ 